\title{
Genome-wide evolutionary characterization and expression analysis of SIAMESE- RELATED family genes in maize
}

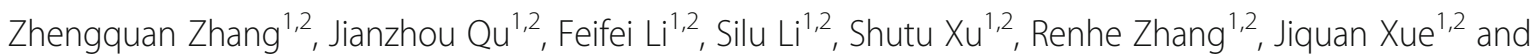
Dongwei Guo ${ }^{1,2^{*}}$

\begin{abstract}
s
Background: The SIAMESE (SIM) locus is a cell-cycle kinase inhibitor (CKI) gene that has to date been identified only in plants; it encodes a protein that promotes transformation from mitosis to endoreplication. Members of the SIAMESE-RELATED (SMR) family have similar functions, and some are related to cell-cycle responses and abiotic stresses. However, the functions of SMRs are poorly understood in maize (Zea mays L.).

Results: In the present study, 12 putative SMRs were identified throughout the entire genome of maize, and these were clustered into six groups together with the SMRs from seven other plant species. Members of the ZmSMR family were divided into four groups according to their protein sequences. Various cis-acting elements in the upstream sequences of ZmSMRs responded to abiotic stresses. Expression analyses revealed that all ZmSMRs were upregulated at 5, 20, 25, and 35 days after pollination. In addition, we found that ZmSMR9/11/12 may have regulated the initiation of endoreplication in endosperm central cells. Additionally, ZmSMR2/10 may have been primarily responsible for the endoreplication regulation of outer endosperm or aleurone cells. The relatively high expression levels of almost all ZmSMRs in the ears and tassels also implied that these genes may function in seed development. The effects of treatments with ABA, heat, cold, salt, and drought on maize seedlings and expression of ZmSMR genes suggested that ZmSMRs were strongly associated with response to abiotic stresses.

Conclusion: The present study is the first to conduct a genome-wide analysis of members of the ZmSMR family by investigating their locations in chromosomes, identifying regulatory elements in their promoter regions, and examining motifs in their protein sequences. Expression analysis of different endosperm developmental periods, tissues, abiotic stresses, and hormonal treatments suggests that ZmSMR genes may function in endoreplication and regulate the development of reproductive organs. These results may provide valuable information for future studies of the functions of the SMR family in maize.
\end{abstract}

Keywords: Endoreduplication, Genome-wide analysis, ZmSMR gene family, Gene expression, Maize, Endosperm, Stress tolerance

\footnotetext{
* Correspondence: gdwei@nwsuaf.edu.cn

${ }^{1}$ Key Laboratory of Biology and Genetic Improvement of Maize in Arid Area of Northwest Region, Ministry of Agriculture, College of Agronomy, Northwest A\&F University, Yangling 712100, Shaanxi, China

${ }^{2}$ Maize engineering technology research center of shaanxi province, Yangling 712100, Shaanxi, China
}

(c) The Author(s). 2020 Open Access This article is licensed under a Creative Commons Attribution 4.0 International License, which permits use, sharing, adaptation, distribution and reproduction in any medium or format, as long as you give appropriate credit to the original author(s) and the source, provide a link to the Creative Commons licence, and indicate if changes were made. The images or other third party material in this article are included in the article's Creative Commons licence, unless indicated otherwise in a credit line to the material. If material is not included in the article's Creative Commons licence and your intended use is not permitted by statutory regulation or exceeds the permitted use, you will need to obtain permission directly from the copyright holder. To view a copy of this licence, visit http://creativecommons.org/licenses/by/4.0/ The Creative Commons Public Domain Dedication waiver (http://creativecommons.org/publicdomain/zero/1.0/) applies to the data made available in this article, unless otherwise stated in a credit line to the data. 


\section{Background}

Cell-cycle regulation is an important mechanism that allows multicellular organisms to adapt to various internal and external stimuli. This process is regulated by key cell-cycle regulatory proteins, including cyclindependent kinases (CDKs) and their corresponding cyclin $(\mathrm{CYC})$ partners $[1,2]$. The primary model of the cell cycle assumes that duplicated DNA undergoes equal division and is distributed into two daughter cells by mitosis and cytokinesis. A modified model assumes that endoreplication or endoreduplication involves multiple rounds of DNA replication without cell division, thereby resulting in polyploidization [3, 4]. However, our understanding of specific regulatory mechanisms for each component of the cell cycle remains limited.

Endoreplication occurs in various organs of approximately $90 \%$ of angiosperms [5]. Endoreplication occurs especially in tissues and cells with rapid proliferation and high rates of metabolic activity [6] such as antipodal cells, synergid cells of female gametophytes, suspensors, cotyledons, filaments, and tapeta [5], plumular axes of seedlings [7], stem and leaf epidermis [8], maize endosperm [4], sorghum endosperm [9], fruit mesocarp [10], and young leaves [11]. Endoreplication can promote petal development in the epidermis of Arabidopsis thaliana [12] and cabbage [8]; it also protects plants against various stressful environmental stimuli such as salt, heat, and cold [13-15]. Arabidopsis is more able to tolerate damage from water deficits when the endoreplication level increases in leaves [13]. Endoreplication is also a common pathway for cell growth during the development of cereal seeds such as rice, wheat, sorghum, and maize. At 8-10 days after pollination (DAP), the middle cells of the endosperm gradually turn to endoreplication. At 20 DAP, the DNA content of these cells reach 96$162 \mathrm{c}$, indicating that these cells may have undergone at least six rounds of DNA synthesis; in terms of the grain development process, the period of endoreduplication and the period of grain filling are almost completely synchronized, indicating a close relationship between kernel formation and endoreplication [16, 17]. In addition, at the late stage of endosperm development, endoreplication is also considered as a signal for the initiation of programmed cells death, which will contribute to the disintegration of endosperm cells and to release the large amount of nucleic acid and phosphoric acid that can be used for embryonic development and germination [18].

Endoreplication is a complex process, and its regulation is mainly achieved via the activity of cyclindependent kinase (CDK). When CDK activity is high, cells tend to undergo normal mitosis; with low activity, the cells transition to endoreplication. The activity of cdk is regulated by a variety of factors; it can be inhibited either by binding to chaperone protein-cyclin (CYC) to produce a heterodimer or by binding to a cyclin kinase inhibitor (CKI). $A-, B$-, and $D-C Y C$ are expressed in maize endosperm, and the peak CDK activities of CycB1;3, D5;1 and D2;1 occur at 11 DAP and are indicative of high mitotic and endoreplication activities [19, 20]. The major CDK inhibitors (CKI) include Wee1 [21] and three CKIs, namely KRP;1, KRP;2 [22], and RBR3 $[23,24]$. The highest expression level of Wee1 was observed during endoreplication, indicating that it may contribute to the inhibition of cell division [25]. KRP;1 and KRP;2 inhibit the related kinase activities of CycA1; 3 and $\mathrm{CycD5}$;1, which involves the regulation of the $\mathrm{S}$ phase [22]. In contrast, RBR1 inhibits RBR3 [23]. The expression of $R B R 1$ is constant and increases during the later stages of endoreplication. RBR3 expression, however, is drastically reduced after commencement of endoreplication, indicating that it is involved in mitosis but that it is not essential to endoreplication. The upregulation of RBR1 during endoreplication implies that it may regulate the conversion of the $\mathrm{G}$ and $\mathrm{S}$ phases during internal endoreplication [23].

Arabidopsis trichomes are considered as an ideal model for studying endoreplication [26]. Low levels of endoreplication can induce trichomes transformation into epidermal cells, even though these have entered the early stages of differentiation; in turn, high levels of endoreplication can induce epidermal cells to form trichomes [27]. The endoreplication level of Arabidopsis trichomes is regulated positively by the SIM gene, and SIM also is the only regulator found to date that exists only in plants [28]. In the Arabidopsis SIM mutation, the trichomes become a multicellular cluster with a singlecell branched shape, and the DNA content in each cell nucleus is significantly reduced [28]. The SIM gene encodes a cyclin kinase repressor (CKI) that initiates mitotic activity toward endoreplication by interacting with CYCD/CDK. The SIM is localized in the nucleus, and it interacts with one or more CYCD/CDDA complexes but not with cyclin CYCB or CDKB [29]. In maize, two SMR proteins have been found that contain the pest domain [30]; however, details concerning the expression and function of these proteins have not been documented.

In the present study, 12 members of the maize $S M R$ gene family were identified by screening the GDB maize database. The protein character, promoter structure, chromosome localization, and protein motif and phylogeny were analyzed, and the results were employed to predict the roles of various cis-acting elements in the promoter regions of the ZmSMR genes in endogenous and stress responses. Finally, the expression differences of these members at the transcriptome level under different endosperm development stages, tissues, abiotic stresses, and hormonal treatments were determined. Collectively, our findings may contribute to our 
understanding of the role of SMR genes in the maize defense response and may also provide valuable information concerning their roles in endoreplication.

\section{Results}

Identification and nomenclature of SMR family members in maize

A total of 12 SMR family members were identified in the maize genome (Additional file 1), hereby designated as ZmSMR1-12 (Table 1). Among the identified ZmSMRs, ZmSMR11 was the largest protein, with 152 amino acids (aa), whereas ZmSMR1 was the smallest, with 90 aa. The molecular weights of the 12 members ranged from 9272.63 Da (ZmSMR2) to $16,404.56 \mathrm{Da}$ (SMR10), with an average of 13,560.16583 Da. Their isoelectric points varied from $5.80 \mathrm{pH}$ (ZmSMR2) to 10.52 $\mathrm{pH}$ (ZmSMR7). Subcellular locations as predicted by Softberry-ProtComp Version 9.0 indicated that $\mathrm{ZmSMR} 4 / 5 / 6$ were present in the nucleus, with scores of 7.6, 7.5, and 8.0, respectively (Table 1 ). In contrast, the predicted position of ZmSMR9 was located in the plasma membrane (score of 8.8). The other SMR members showed extracellular localization with relatively low scores. In addition, ZmSMR4/5/6 contained 2-4 skn-1_ motifs, the decisive elements for endosperm development [31], which were not found in the sequence of ZmSMR9 (Additional file 3).

\section{Regulatory elements in the promoter sequences of ZmSMRs}

Cis-elements in the promoter regions are required for temporal, spatial, and cell-specific control of gene expression [32]. The upstream sequences $(1.5 \mathrm{~kb})$ of the 12 ZmSMRs were submitted to the PlantCARE database, PlantPan 2.0 and RegSite Database of Plant Regulatory
Elements-Softberry to identify cis-acting elements. Additional files 2 and 3 list 47 endoreduplication-related elements (E2F elements) [33] situated within the promoter regions of all of ZmSMRs except for ZmSMR6. Fifteen 5'UTR Py-rich stretch elements, which positively influence the overall expression level [34], were present in the promoter regions of eight $Z m S M R$ genes. Eleven promoters contained 1-6 Skn-1_motifs involved in endosperm development [31]. As for salt- or drought stress-related elements, the MBSI motif was predicted only in the promoter of ZmSMR4, and nine promoters contained MBS [35]. The low-temperature-responsive element (LTR) [36] and heat-shock element (HSE) [37] were found in six and four genes, respectively, and only the ZmSMR4 and ZmSMR5 had both elements (Additional file 3). Two ABA-responsive elements, ABREs and CE3 [38], were detected in twelve and three ZmSMR genes, respectively.

\section{Chromosomal distribution and gene duplication of the SMR gene family in multiple species}

The physical map positions of the 12 ZmSMRs on ten maize chromosomes were identified by BLAST against the maize genome. The 12 genes were distributed nonrandomly across 7 out of the 10 maize chromosomes, except for chromosomes 7, 8, and 10 (Fig. 1). Chromosome 9 contained several ZmSMRs comprising three members of ZmSMR3/4/5, whereas chromosomes 1, 3, and 5 each harbored two ZmSMR genes, and chromosomes 2, 4, and 6 carried only one member, namely ZmSMR12/7/8.

Segmental- or whole-genome duplications are common phenomena in plant genome evolution, resulting in the expansion and diversification of many gene families and the evolution of the organism [39]. The maize

Table 1 List of identified SMR genes in Zea mays L. along with their corresponding proteins' information

\begin{tabular}{|c|c|c|c|c|c|c|c|}
\hline Gene & $\begin{array}{l}\text { Gene accession. } \\
\text { No. }\end{array}$ & $\begin{array}{l}\text { Chromosome } \\
\text { No. }\end{array}$ & $\begin{array}{l}\text { CDS length } \\
\text { (bp) }\end{array}$ & $\begin{array}{l}\text { Protein } \\
\text { length(aa) }\end{array}$ & $\begin{array}{l}\text { Molecular } \\
\text { weight(Da) }\end{array}$ & $\begin{array}{l}\text { Isoelectric } \\
\text { point(ph) }\end{array}$ & $\begin{array}{l}\text { Sub-cell location } \\
\text { (scores) }^{a}\end{array}$ \\
\hline ZmSMR1 & Zm00001d042902 & chr3 & 273 & 90 & 9272.63 & 9.10 & Extracellular (2.6) \\
\hline$Z m S M R 2$ & Zm00001d012956 & chr5 & 288 & 95 & $10,211.6$ & 5.80 & Extracellular (2.7) \\
\hline$Z m S M R 3$ & Zm00001d046896 & chr9 & 426 & 141 & $14,441.9$ & 9.76 & Extracellular (2.4) \\
\hline$Z m S M R 4$ & Zm00001d047159 & chr9 & 381 & 126 & $13,850.9$ & 9.83 & Nuclear(7.6) \\
\hline$Z m S M R 5$ & Zm00001d048533 & chr9 & 411 & 136 & $14,773.9$ & 9.33 & Nuclear(7.5) \\
\hline ZmSMR6 & Zm00001d029820 & chr1 & 411 & 136 & $14,719.9$ & 9.66 & Nuclear(8.0) \\
\hline$Z m S M R 7$ & Zm00001d049769 & chr4 & 321 & 106 & $11,526.1$ & 10.52 & Extracellular (2.5) \\
\hline$Z m S M R 8$ & Zm00001d036683 & chr6 & 456 & 151 & $15,494.8$ & 8.92 & Extracellular (2.5) \\
\hline$Z m S M R 9$ & Zm00001d031546 & chr1 & 381 & 126 & $13,246.0$ & 10.25 & Plasma membrane(8.8) \\
\hline$Z m S M R 10$ & Zm00001d015140 & chr5 & 456 & 151 & $16,404.56$ & 9.00 & Extracellular (2.4) \\
\hline$Z m S M R 11$ & Zm00001d039827 & chr3 & 459 & 152 & $15,689.8$ & 10.51 & Extracellular (2.3) \\
\hline ZmSMR12 & Zm00001d006060 & chr2 & 384 & 127 & $13,089.9$ & 10.34 & Extracellular (2.5) \\
\hline
\end{tabular}

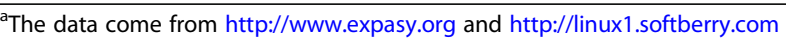




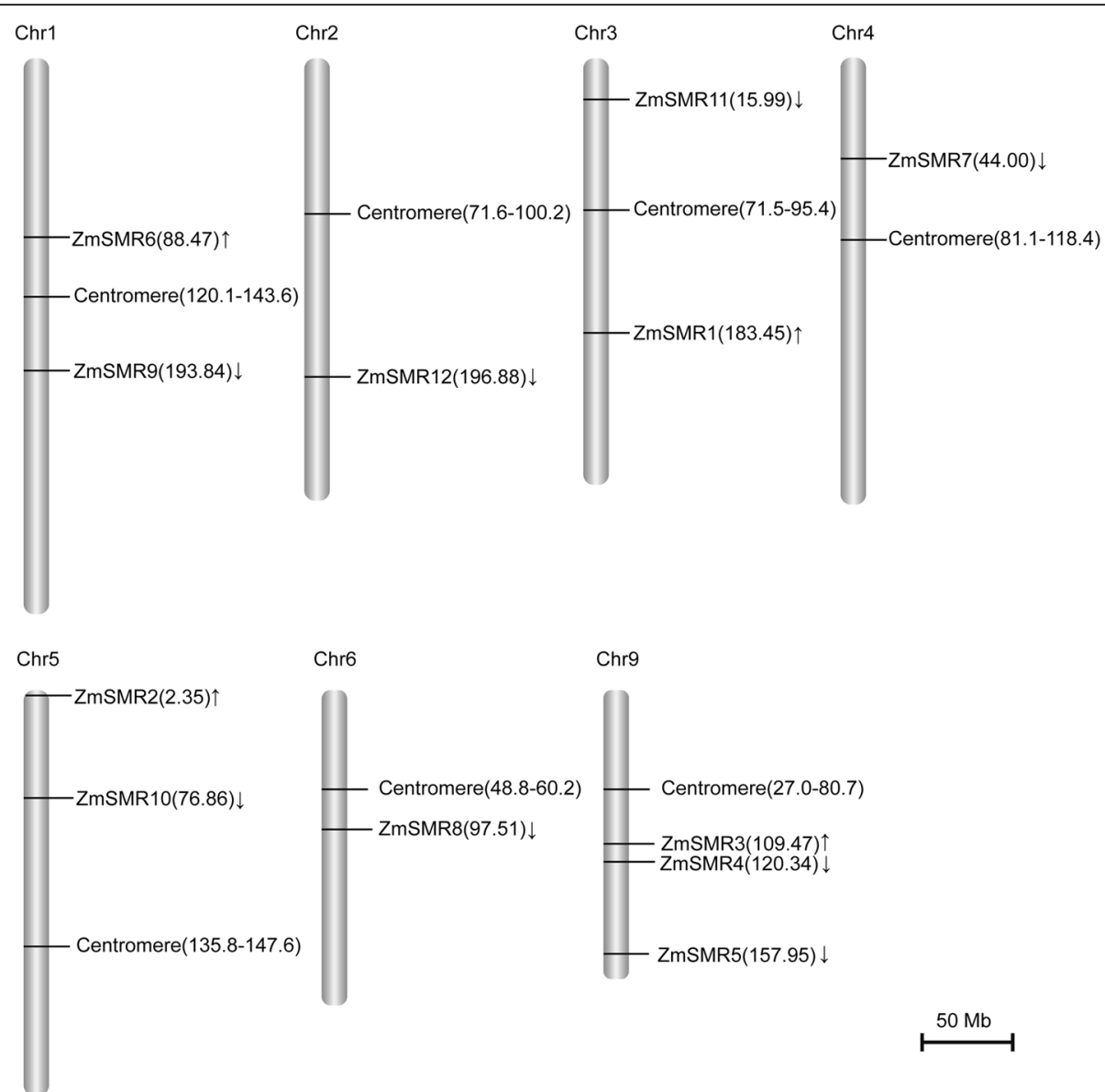

Fig. 1 Chromosomal distribution of 12 ZmSMR genes. The chromosome number is indicated on the top of each chromosome. The value of the position of each gene and centromere on the chromosome is labeled on the right side of the chromosome bar. The scale of the chromosome length is $50 \mathrm{px}=50 \mathrm{Mb}$

genome has undergone two rounds of genome duplication, where its size has expanded dramatically (to 2.3 gigabases) over the last three million years via a proliferation of long-terminal-repeat retrotransposons [40]. Among ZmSMR genes, only ZmSMR3 and ZmSMR8 were found to be a pair of syntenic genes; these were located on chromosomes 9 and 6, respectively (Fig. 2 and Additional file 4). Similarly, only two SMR genes were found to be syntenic genes in Glycine max, Sorghum bicolor and Setaria italica. In contrast, there were six (55\%) and nine (47\%) syntenic genes in Populus trichocarpa and Brachypodium distachyon, respectively. It is worth noting that there were 23 pairs of syntenic genes in maize and B. distachyon, O. sativa, S. bicolor, and $S$. italica (Fig. 2 and Additional file 4).

\section{Phylogenetic and conserved-motif analysis of ZmSMRs}

Phylogenetic reconstruction indicated that the 12 ZmSMRs could be clustered into four distinct subgroups, named A to D (Fig. 3). Subgroup C contained the maximum number (four members) of ZmSMRs,
ZmSMR7/9/11/12, followed by subgroups A and D with three members each, and subgroup B with two genes. Furthermore, the ZmSMR3 and ZmSMR8 belonged to subgroup B showed bootstrap values of 98 , with alignment identity of $74.05 \%$ (Additional file 5), and they were a pair of synteny genes (Fig. 2). For ZmSMR4/6/5, which were predicted to be located in the nucleus (Table 1 ), members of subgroup A were generally similar in terms of protein length, molecular weight, and isoelectric point.

Six conserved motifs were detected in the 12 ZmSMRs by MEME online search software (Fig. $3 \mathrm{~b}$ and Additional file 6). The ZmSMRs within the same subgroup possess similar motif compositions. For example, members of subgroup $\mathrm{C}$ contained exactly the same motif composition of Motif6-Motif2-Motif4-Motif1-Motif3. Subgroup A harbored motif 5 at its N-terminal, whereas motif 6 was only observed in subgroups B and C (Fig. 3). The results revealed that all members shared motifs $1 / 2$ / $3 / 4$, except for ZmSMR2 that only harbored motifs 1 and 2 . In addition, motifs $1,2,4$, and 5 were identified 


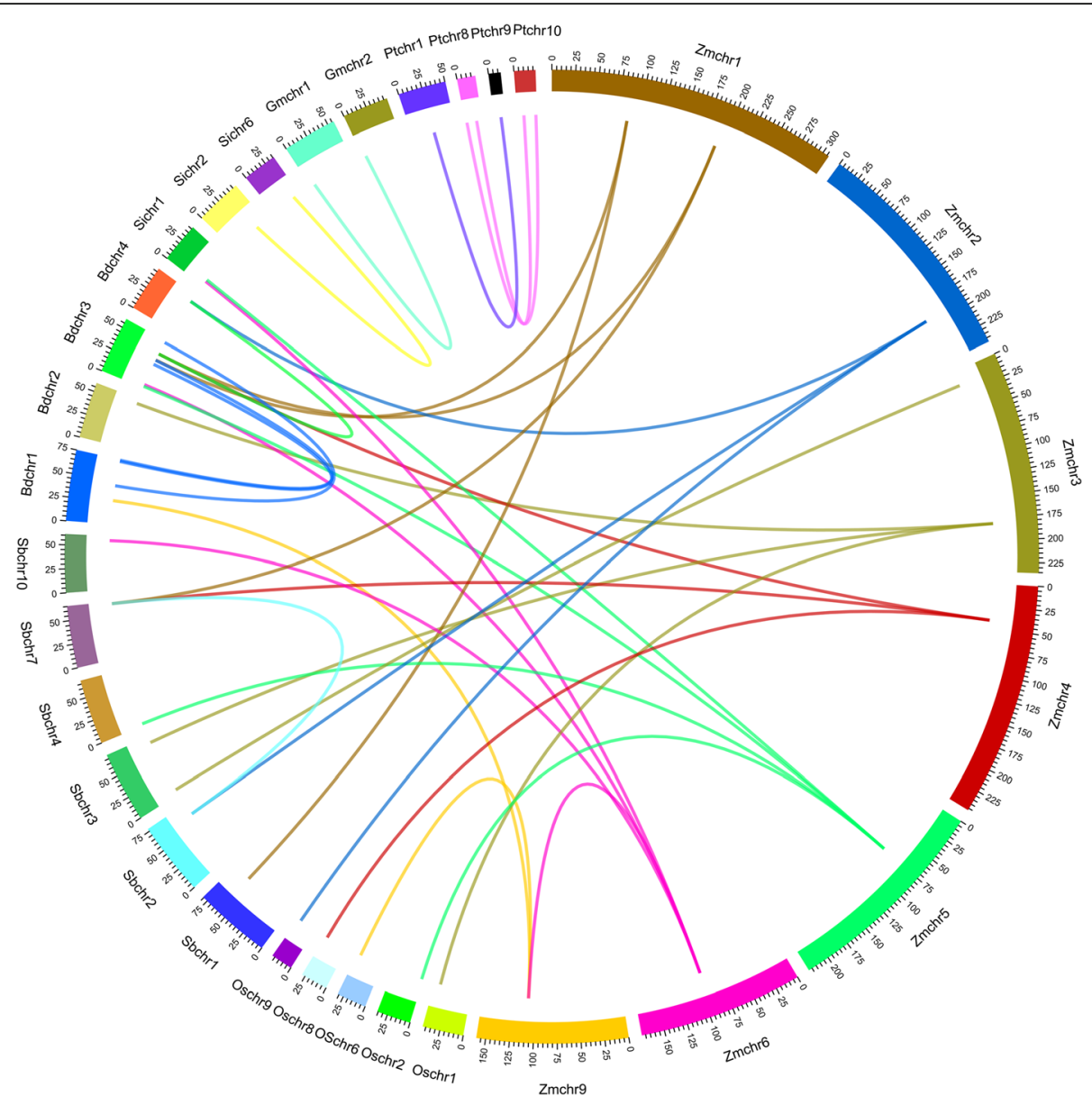

Fig. 2 Distribution of genome syntenic and intergenomic synteny SMR gene pairs in multiple species. Analyzed species are Zea mays (Zmchr), Brachypodium distachyon (Bdchr), Oryza sativa (Oschr), Sorghum bicolor (Sbchr), Setaria italic (Sichr), Populus trichocarpa (Ptchr) and Glycine max (Gmchr). The scale line of the chromosome size is also identified in the figure

by Churchman [30] and are herein described as motifs 2, 4, 1, and 3, respectively. Motif 1 of the ZmSMR family was a domain of the rice EL2 protein that interacts with cyclin [41] and is similar to motif 3 of the CDK-inhibitory ICK/ KRP proteins (Fig. 3) [42]. Motif 2 contained a minimum site TP that is phosphorylated by CDKs [43]. This pair of amino acid residues was the most conserved during the evolution of ZmSMR proteins. Motif 4 is rich in proline and consists of a typical PXXP structure followed by one or several amino acid residues. This domain is a proteininteraction site that allows proteins to interact with certain ligands by forming PPII helices [44].

To further examine the evolutionary relationships among the SMR proteins from different species, an unrooted phylogenetic tree was constructed using the full-length SMR proteins from monocotyledons of $Z$. mays, Oryza sativa, S. bicolor, S. italica, B. distachyon, the dicotyledon of A. thaliana, G. max, P. trichocarpa, and the musci of $P$. patens. All SMRs from the above species were divided into seven subgroups. No SMR homologous genes were detected in the algae. However, a total of ten SMRs were detected in P. patens, nine of which were clustered in Group VI, and the other members of this subgroup were GmSMR1, SiSMR3, PtSMR8, BdSMR17 and OsSMR6. Thus, these five proteins may be very conserved during the evolution of their respective species, and that the ancestors of the SMR family first appeared in the $P$. patens.

All subgroups contained monocotyledons or dicotyledon species except for Group VI (Fig. 4). In addition, the members of the four subgroups, A, B, C, and D were distributed among Groups III/I/II. In total, 18 pairs of SMRs from different species were clustered as pairs (Fig. 4). The proteins of ZmSMR7 and BdSMR13, ZmSMR10 and BdSMR14, ZmSMR5, and BdSMR15 were highly similar, indicating that some consensus in proteins may have existed before the divergence of $B$. distachyon and $Z$. mays. It was worth noting that OsSMR11 and the PpSMR8 were clustered into one pair of genes, indicating that OsSMR11 was very conserved during rice evolution. 


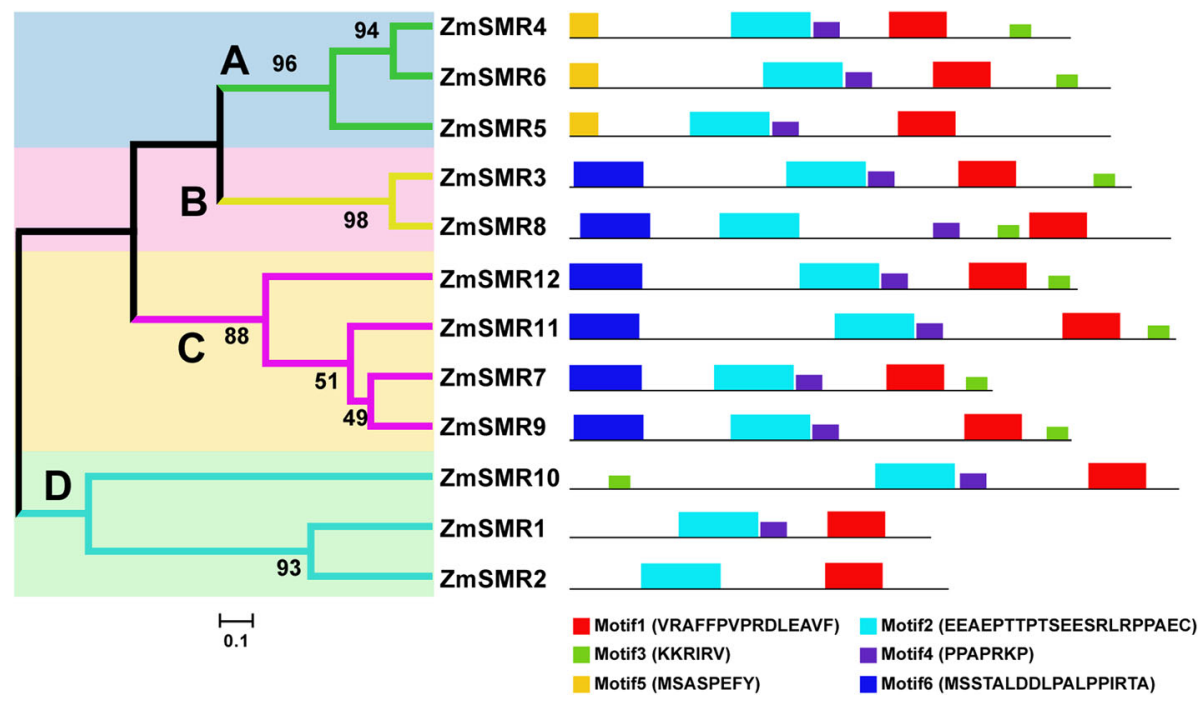

Fig. 3 Phylogenetic analysis and motif composition of ZmSMR proteins. The phylogenetic tree was constructed using MEGA6 software by the Neighbor-joining method with 1000 bootstrap iterations based on the 12 full-length amino acids of ZmSMR, and the proteins were clustered into four subgroups ( $A, B, C, D$ ) marked with different background to facilitate subfamily identification. Schematic representation of the conserved motifs in the ZmSMR proteins was analyzed by MEME. Each motif is represented by a colored box numbered at the bottom, and the consensus sequences of each motif are also shown. The details of sequence logo of the motifs are given in Additional file 6

Relationship between grain-filling rate and expression of ZmSMRs during maize endosperm development

Maize embryo tissue is relatively young, and endoreduplication activity often occurs in this rapidly developing and highly metabolically active tissue [6]. To test the role of ZmSMRs in the development of maize embryos, we examined the grain-filling rate of the inbred line, B73, every 5 days from 5 to 35 DAP. The grain-filling rate increased sharply at 5-10 DAP and 15-20 DAP, coinciding with the beginning and active periods of endoreduplication, respectively. In contrast, a moderate trend in grain-filling rate was observed from 10 DAP to 15 DAP when endoreduplication activity was initiated (Additional file 10). Endoreduplication levels were lower during the programmed cell death (PCD) stage, which was observed at 20 DAP [45], occurring simultaneously with the gradual reduction in the grain-filling rate (Fig. 5a).

Gene expression may provide a value clue for exploring gene function [46]. Here, quantitative real-time PCR (qRT-PCR) was used to analyze the expression profiles of the $12 \mathrm{ZmSMRs}$ in the endosperm every 5 days from 5 to 35 DAP (Fig. 5b). The univariate linear regression statistics of $Z m S M R$ gene expression and grain filling rate showed that the expression level of almost all genes was negatively correlated with the rate of grain filling, with $Z m S M R 1 / 12$ reaching an extremely significant level $(P<0.01)$ and $Z m S M R 6$ reaching a significant level $(P<$ 0.05). Multiple linear regression showed a correlation coefficient of 0.977 , which was extremely significant $(P<$ 0.01) (Table 2). The $12 \mathrm{ZmSMR}$ genes were significantly upregulated at 5 DAP, 20 DAP and 25 DAP, coinciding with a dramatic increase in grain filling rate. These genes have both motif 1 and motif 2 . Motif 1 may be the CDK-inhibitory ICK/KRP protein (Fig. 3) [42]. The TP site of Motif 2 is involved in CDK regulation of the cell cycle [43].

The expression patterns of genes can provide a preliminary indication for revealing its function [47]. Expression profiling of $12 \mathrm{ZmSMR}$ genes in maize endosperm from 5 to 35 DAP was performed. In general, the expression pattern of ZmSMR genes exhibited a "W" shape, wherein they were upregulated at 5 DAP, 20 DAP, and 25 DAP, 35DAP, which coincided with the rapid increase in the grain-filling rate that in turn contributed to the development of the maize endosperm.

ZmSMR9/11/12 was upregulated at 10 DAP, whereas $Z m S M R 2 / 10$ was highly expressed at 15 DAP. These two groups of genes exhibited similar expression patterns, and they belonged to subgroups $C$ and $D$, respectively. The endoreduplication had already started in the endosperm of 5 DAP due to the appearance of $12 \mathrm{C}$. The highest ploidy level was reached at 20-30 DAP, maintaining $96 \mathrm{C}$ (Additional file 10). Our results indicated that ZmSMRs, although negatively correlated with grain filling, may promote endoreduplication during the 5,20 , and 25 DAP phases.

\section{Expression patterns of ZmSMRs in diverse tissues of maize}

Transcript levels of all ZmSMRs were examined in diverse maize tissues, including root, leaf, internode, node, ear, and tassel (Fig. 6). High expression levels of all 


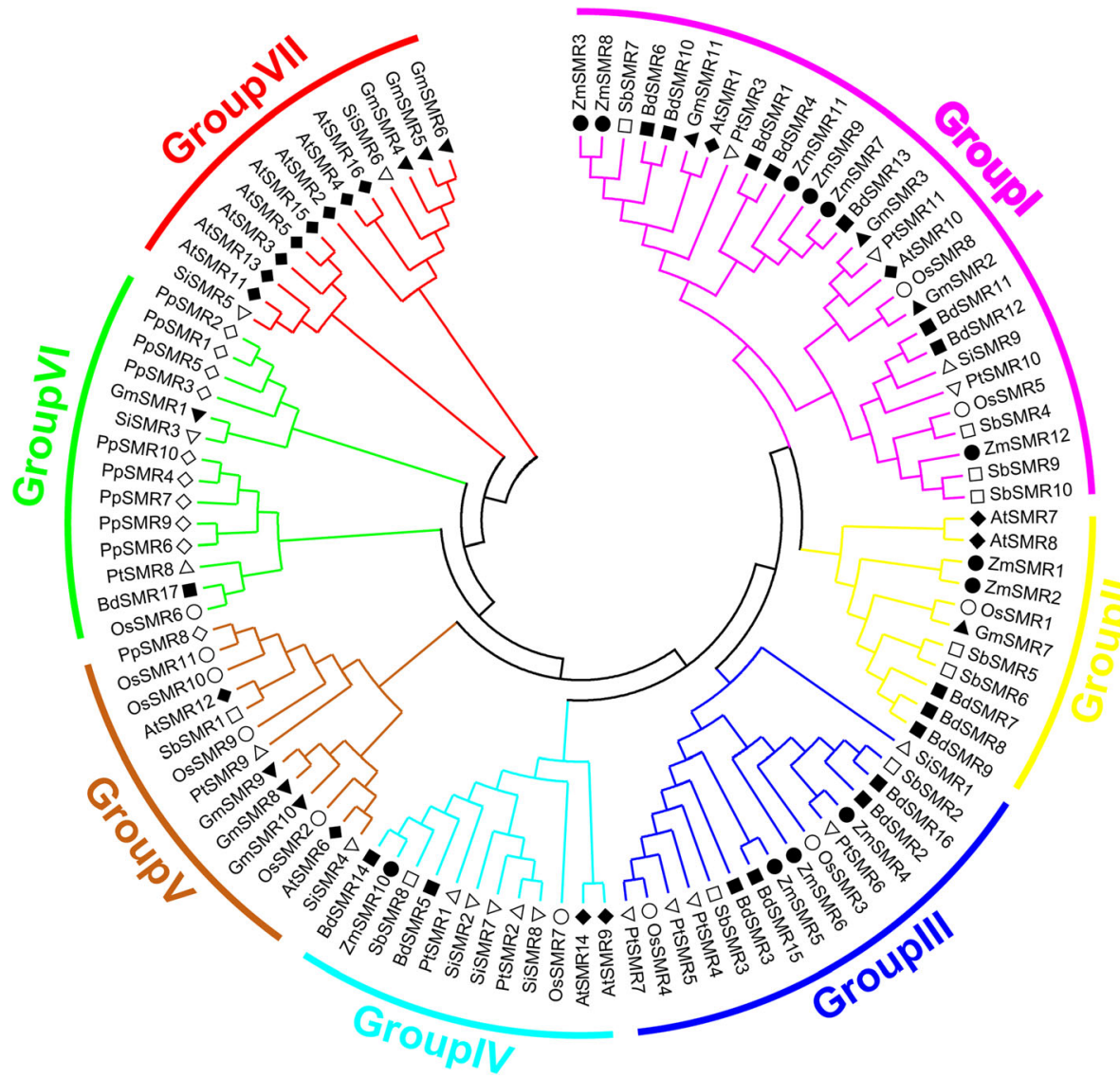

Fig. 4 Phylogenetic tree of SMR proteins from Z. mays, Arabidopsis, O. sativa and other species. The complete amino acid sequences of $12 Z$. mays, 28 Arabidopsis, 17 O. sativa and 71 SMR proteins from other species were aligned by ClustalW, and the phylogenetic tree was constructed using Molecular Evolutionary Genetics Analysis 6 (MEGA6.06) by the Neighbor-joining method with 1000 bootstrap replicates. Each species' gene is labeled with a different symbol. ZmSMR represents Z. mays SMR; AtSMR represents A. thaliana SMR; OsSMR represents O. sativa SMR; BdSMR represents B. distachyod SMR; SiSMR represents S. italica SMR; SbSMR represents Sorghum bicolor SMR; GmSMR represents Glycine max SMR; PtSMR represents Populus trichocarpa SMR; PpSMR represent Physcomitrella patens SMR. The phylogenetic tree was divided into six phylogenetic groups as Group I-VII using different colors

$Z m S M R s$ were observed in the ears and tassels. Therein, eight genes (ZmSMR1, ZmSMR2, ZmSMR3, ZmSMR4, ZmSMR6, ZmSMR8, ZmSMR9, and ZmSMR11) were expressed the highest in ears; one gene (ZmSMR7) had its strongest expression in tassels. $Z m S M R 12$ were expressed predominantly in roots, and ZmSMR5/10 occurred predominantly in leaves. The mRNA accumulation levels of $Z m S M R 6 / 10 / 11$ in leaves, ears, and tassels were similar. Most genes, especially the ZmSMR1/3/6/9/ $10 / 11 / 12$, were expressed relatively lower, in nodes and internodes. The expression levels of ZmSMR8 and $Z m S M R 2 / 4 / 5$ were barely detected in leaves and roots, respectively. ZmSMR3 and ZmSMR8, which were clustered based on protein motif analysis (Fig. 3), shared a similar expression pattern. The tissue-specific expression patterns of $Z m S M R s$ indicate that endoreduplication commonly occurs in various maize tissues, especially in the ears, tassels, and leaves.

\section{Expression profiles of ZmSMRs in response to abiotic stresses}

Endoreplication is affected by the environment and by plant hormones and has been reviewed by Barow [15]. The ZmSMRs contain a variety of cis-acting elements, and these are all expressed in the leaves. Therefore, we investigated the responses of these genes in the leaves under multiple abiotic stress conditions, including ABA $(100 \mu \mathrm{M})$, heat $\left(42^{\circ} \mathrm{C}\right)$, cold $\left(4^{\circ} \mathrm{C}\right)$, salt $(200 \mathrm{mM} \mathrm{NaCl})$, and drought (20\% PEG) (Fig. 7). The qRT-PCR indicated that the increased mRNA levels of ZmSMR3, ZmSMR11, and $Z m S M R 12$ were induced by ABA stress in a timedependent manner. However, ZmSMR4, ZmSMR5, ZmSMR6, ZmSMR7, and ZmSMR8 were significantly upregulated at the 2-h and 24-h time points and were downregulated at the 6-h and 12-h time points (Fig. 7). For the heat and cold treatments, ZmSMR5, ZmSMR7, and $Z m S M R 11$ were differentially expressed at specific 


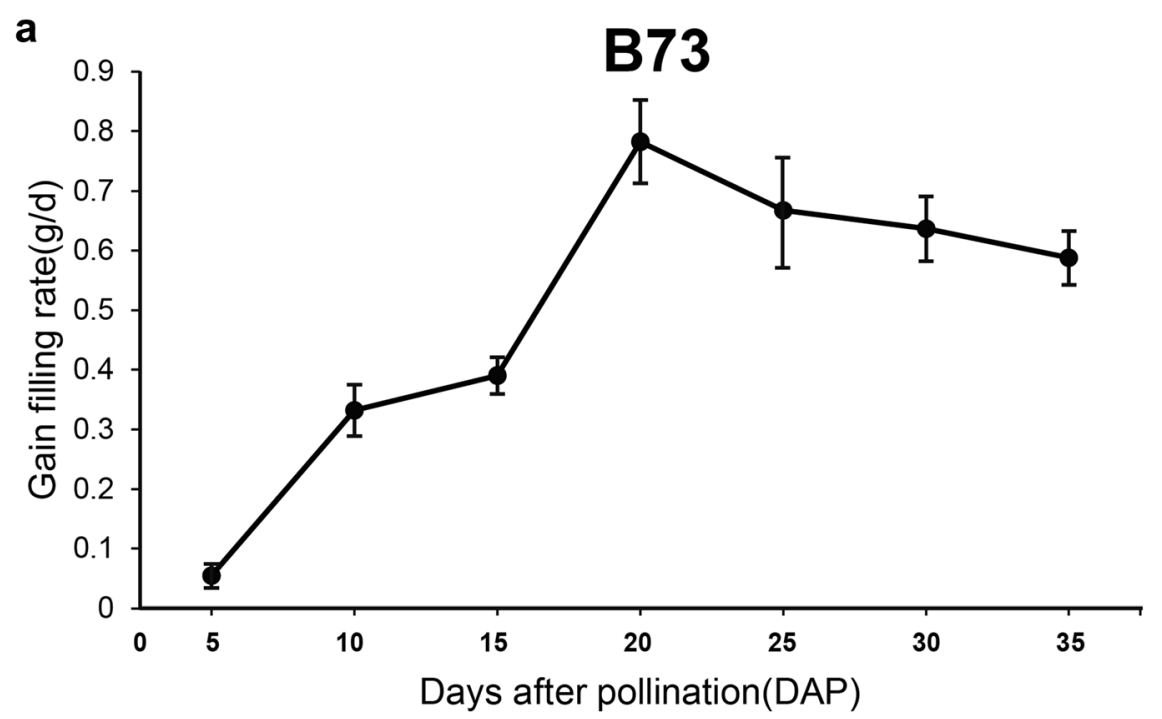

b

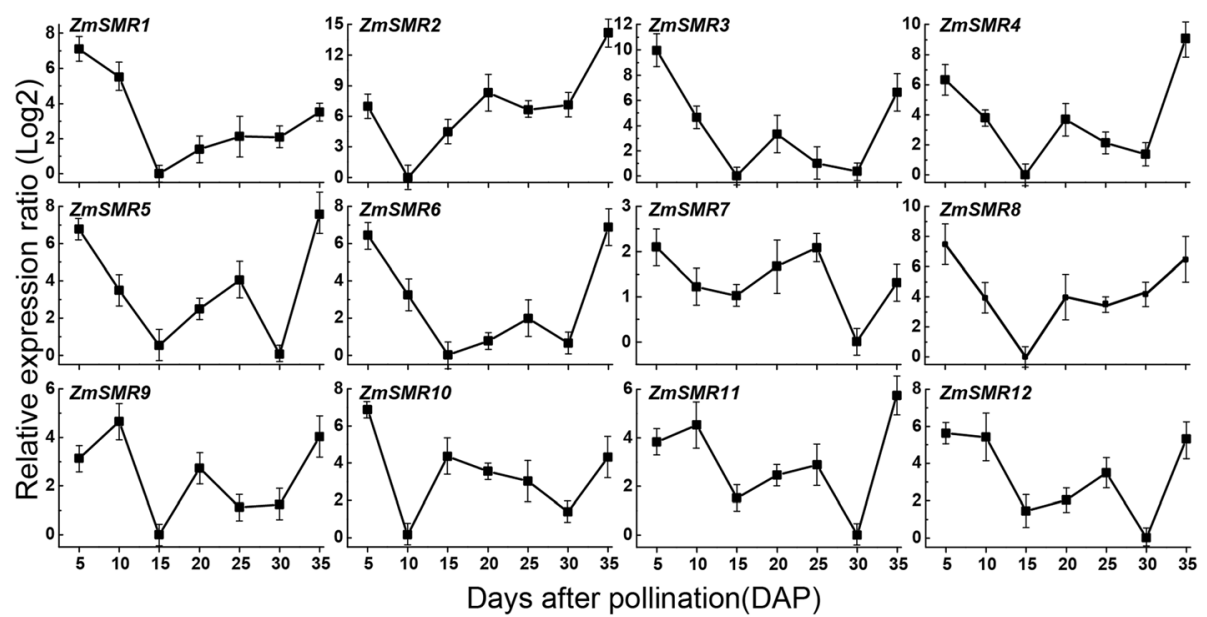

Fig. 5 Grain-filling rate and expression profile of the ZmSMR family genes in maize endosperm. a The grain-filling rate was measured from 5 to 35 DAP every 5 days. The trendline was drawn through the weight increase of hundred-grain weight every 5 days. $\mathbf{b}$ The maize endosperm samples were taken every 5 days from 5 to 35 DAP. Relative expression ratios in these sample were calculated with reference to the sample in which the respective transcript exhibited the lowest expression. The relative expression values were log2 transformed. The qRT-PCR data were normalized against the expression of Actin as an internal control. Error bars indicate standard deviations. The names of the genes are written in the upper right corner of each bar diagram

time points. In contrast, the expression patterns of the other genes were highly similar. ZmSMR3, ZmSMR4, and ZmSMR12 were downregulated at all time points. However, ZmSMR2 was upregulated after the application of heat and cold stress. For salt and drought stress, $Z m S M R 4$ and ZmSMR8 were upregulated from $12 \mathrm{~h}$ to $48 \mathrm{~h}$ after stress application, and then were downregulated at $72 \mathrm{~h}$ after exposure to stress. ZmSMR1 was significantly upregulated at all time points during stress application. The responses of all ZmSMR genes to salt and drought were highly similar at all four time points (Fig. 7). This conformity may be due to cis-acting elements that are involved in salt and drought stresses; both MBSI and MBS [35]. These results indicate that the $Z m S M R$ genes might be involved in stress response to adverse environmental conditions.

\section{Discussion}

The SIM was first isolated in A. thaliana and was determined to have dual functions as a suppressor of mitosis and an elicitor for endoreplication [29]. In addition, the SIM is the only plant factor that has been reported to date that promotes the transformation from mitosis to endoreplication. The tissue-specific expression pattern 


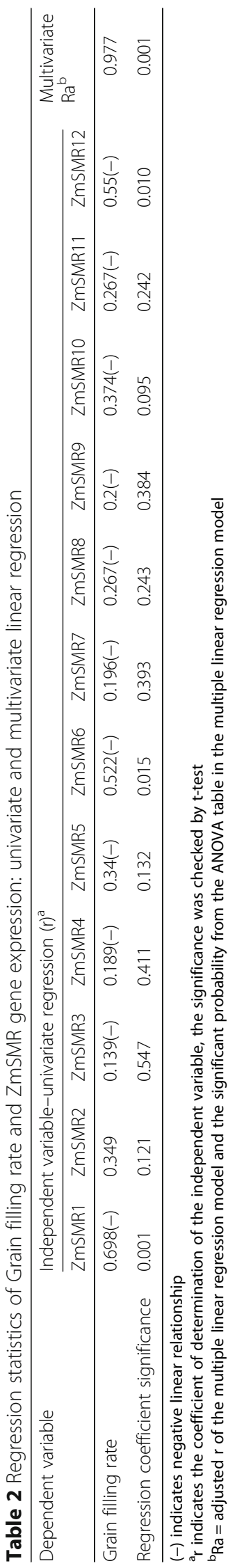




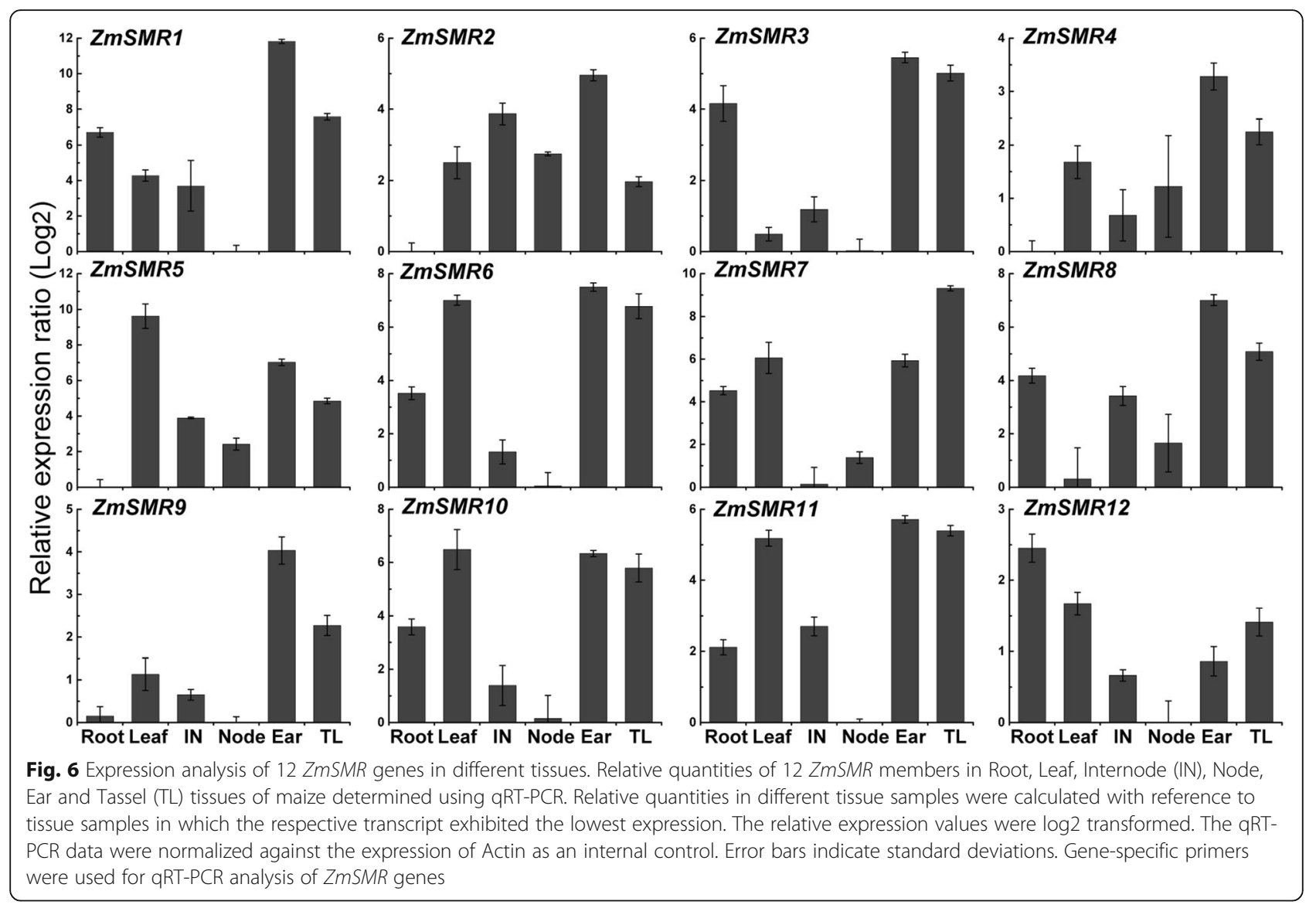

of the SIM has been studied in A. thaliana, and a several bioinformatic analyses of the $S M R$ family have been reported [30], whereas SMR genes have not been well studied in maize.

In the present study, $12 \mathrm{ZmSMR}$ genes were identified in the maize genome via a homology-search method using Arabidopsis SIM/SMR aa sequences as the query [48]. These $12 \mathrm{ZmSMR}$ genes showed significantly different isoelectric points and marked variations in their predicted sub-cellular locations (e.g., ZmSMR4/5/6 was predicted to be situated in the nucleus with a high prediction score, Table 1). Moreover, the $12 \mathrm{ZmSMR}$ genes were nonuniformly distributed across 7 out of the 10 chromosomes of maize (Fig. 1). The observed differences in the characters of the ZmSMR family members and $Z m S M R 4 / 5 / 6$ may reflect their distinct roles in initiating endoreplication.

In previous studies, three motifs were identified in the Arabidopsis SMR family [43], whereas in another study, five motifs were described by aligning the putative protein product of the SIM reading frame and related plant proteins from S. lycopersicum, S. tuberosum, Z. mays, $O$. sativa, P. tremula, and G. $\max [30]$. However, in this study, six motifs were identified in the ZmSMRs. The order of these motifs on the ZmSMR protein sequences was basically the same, except for ZmSMR10, in which motif 3 was located at the N-terminal. It was worth noting that only ZmSMR10 was upregulated at 15 DAP, indicating that the order of motifs may also affect the transcription of the entire gene. However, ZmSMR1 and ZmSMR2 did not contain motif 3. The phylogenetic analysis showed that ZmSMR1/2/10 belong to the same subgroup (Fig. 3), thereby suggesting similar functions, and that a change in the position of motif 3 within the polypeptide may lead to a loss of function. Furthermore, the two new motifs 5 and 6 were detected only in maize. Both were located at the $\mathrm{N}$-terminal and contained aa residues with MS in order. Motif 5 (MSASPEFY) was also detected consistently as domain 12 in the EL2 protein in rice, which was a plant-specific cyclin dependent kinase inhibitor [41], suggesting that motif 5 in ZmSMR4/5/6 may play an important role in the transformation from mitosis into endoreplication. The expression patterns of $Z m S M R$ genes in endosperm showed they were upregulated at 5 DAP, 20 DAP, and 25 DAP, 35 DAP, thereby implying their importance during the initiation and progression of endoreproduction. Studies have shown that the nucleus size from the central endosperm to the aleurone layer of maize gradually decreases, indicating that the internal replication 

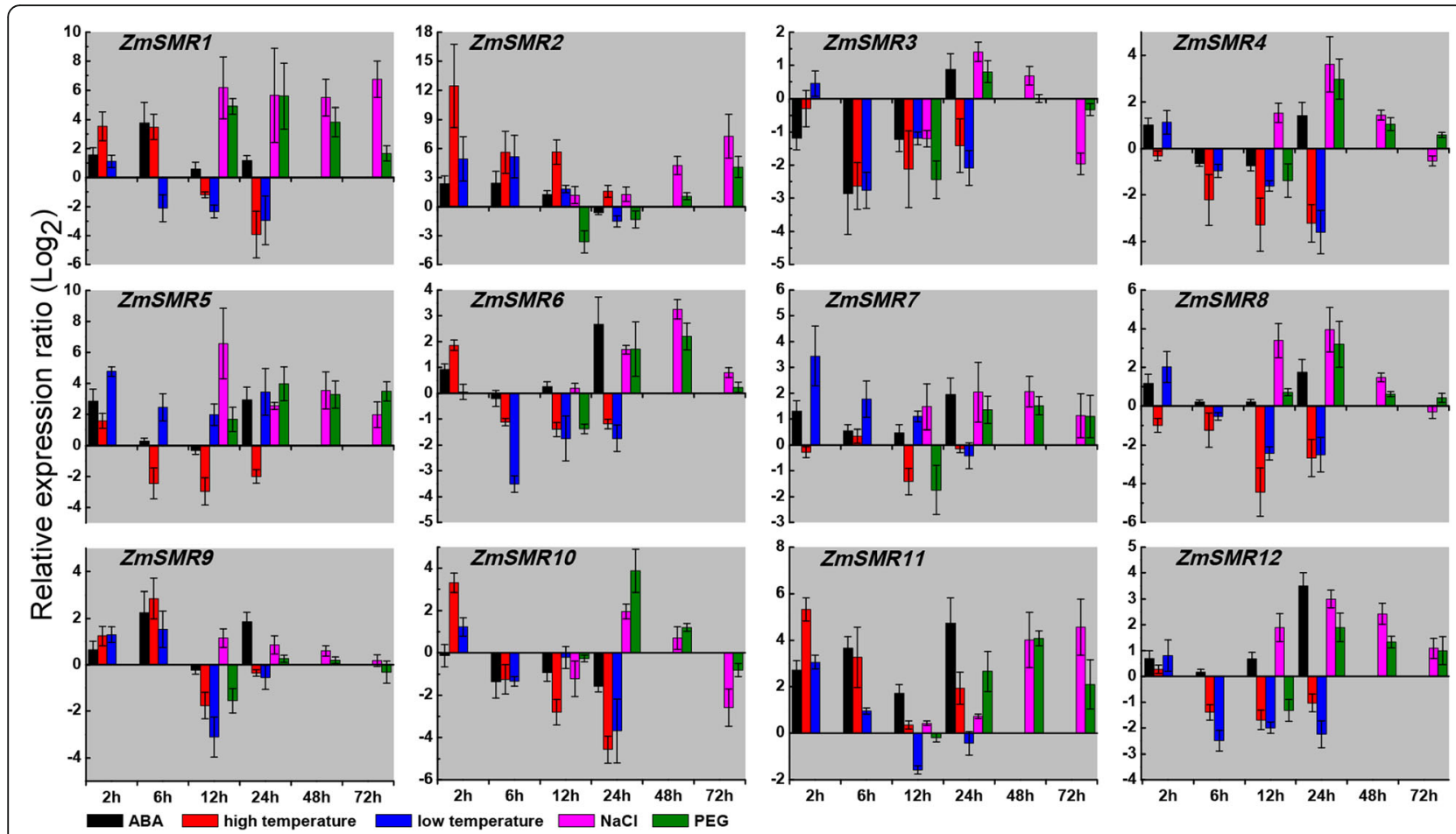

Fig. 7 Expression profiles of ZmSMR genes under various abiotic conditions. The $x$-axes represent treatment time, and $y$-axes indicate scales of relative expression levels. Three-leaf maize seedlings were treated with $4^{\circ} \mathrm{C}$ (low temperature), $42^{\circ} \mathrm{C}$ (high temperature), or $100 \mu \mathrm{M}$ of $\mathrm{ABA}$ for 24 $\mathrm{h}$, or with $20 \%$ PEG and $200 \mathrm{mM}$ of $\mathrm{NaCl}$ for $72 \mathrm{~h}$. Mixed leaves of three seedlings as well as the untreated leaves were collected after treatment for $2,6,12,24,48,72 \mathrm{~h}$, where the untreated leaves were regarded as the control check (CK). The relative expression values were log2 transformed. The qRT-PCR data were normalized against the expression of Actin as an internal control. Error bars indicate standard deviations

process develops from the endosperm center to the edge along a gradient [4]. The expression of ZmSMRs is timespecific, which may be due to expression in different endosperm subaleurone domains at different stages of endosperm tissue development. The endosperm cells in the center of 8-10 DAP grains gradually shifted from mitosis to internal replication cycles [4, 18]. In addition, all ZmSMRs were highly expressed during 5-10 DAP; $Z m S M R 9 / 11 / 12$ was especially up-regulated during this period, indicating that these three genes may play a major role in initiating endoreplication of endosperm central cells. At 16 DAP, most cells in the endosperm entered endoreplication [49]. At the same time, the mitotic index of the endosperm had fallen to less than $1 \%$. The qRT-PCR results showed that ZmSMR2/10 were up-regulated at 15 DAP, indicating that they may be mainly responsible for the endoreplication regulation of outer endosperm or aleurone cells. It has been reported that expression of CYCB1; 1 and CYCB1; 2 are reactivated in the trichome of SIMESE-mutated Arabidopsis [30]. In the developing maize endosperm, the expression of CDKA protein is relatively stable during the cell cycle, while the expression of CDKB associated with the $M$ phase is decreased [21]. Therefore, ZmSMRs as CKIs may inhibit the expression of CYCB1;1 and CYCB1;2 by degrading $C D K B$ and thereby maintain the cells in a state of endoreplication. Some maize endosperm cells enter the PCD stage at 20 DAP [45]; however, endoreduplication can mark cells for programmed death [19]. At 35 DAP, all ZmSMRs were upregulated. These genes may thus possibly regulate the transition of endosperm cells from the endoreplication stage to the apoptosis stage, thereby reducing the grain filling speed. This may also be due to the gradual drying of the endosperm during this period, which causes the up-regulation of ZmSMRs expression in ABRE-rich elements. However, further details concerning the mechanisms underlying maize endoreplication require additional investigation.

SIM was first detected in the trichomes of A. thaliana, and it has also been studied in the roots, rosettes, stems, siliques, and flowers and is particularly upregulated in the roots and stems [30]. Analysis of tissue expression indicated that all ZmSMR genes were upregulated in the ears and tassels and thus may be involved in regulating the development of reproductive organs. In contrast, ZmSMR2/4/5/9 were downregulated in the roots, which may be due to the differentiation of their functions compared to the SIM gene. SMR3 and SMR8 belonged to Group B and showed similar expression profiles, suggesting that their functions may be highly similar. 
Endoreplication is not only related to plant growth and development $[6,8,11,12]$ but also to various abiotic stresses $[13,14]$. Moreover, almost all the promoter regions of ZmSMRs have the ABRE cis-elements in response to ABA stress [38] and E2F cis-elements that regulate endoreplication [33]. In this study, five stress conditions were examined (ABA, heat, cold, salt, and drought). The observed upregulation of $\mathrm{ZmSMR}$ genes was apparently in response to at least one of the stress conditions. ABA has recently been reported to play crucial roles in response to abiotic stresses, such as drought or salinity [50] that cause the G1-to-S transition to be impaired, thereby slowing DNA replication and/or delaying entry into mitosis [41]. CKI is a candidate protein involved the molecular mechanisms that link the stress perception directly to the cell-cycle machinery [41]. SMR, as a kind of CKI, may also be associated with response to environmental stresses. In this study, ZmSMR9, ZmSMR11 and ZmSMR12 genes were induced under ABA stress, and they may specifically express in the center of the endosperm tissue, indicating that these three genes may play direct or indirect roles in response to ABA stress and in seed development. Phylogenetic analysis also showed that they all belonged to subgroup $\mathrm{C}$, indicating that their functions were more conservative. In addition, the tendency of ZmSMRs to be up- or down-regulated under hot and cold stress was very similar to ABA stress, indicating that induction of ZmSMRs by hot and cold occurred in an ABAdependent manner. The expression of ZmSMR5 and ZmSMR7 under cold treatment had been induced and was the same as the response of the rice EL2 gene to cold stress [41]; EL2 was identified as a cell-cycle regulatory gene related to the SIM gene of Arabidopsis thaliana. These results show that overexpression of ZmSMR5/7 might be responsible for cold tolerance. Endoreplication can also allow cells to adapt to salt stress [13], and Churchman confirmed this conclusion by reporting that Arabidopsis $S M R 3 / 4 / 5$ was upregulated by salt stress [41]. In addition, drought can inhibit endoreplication in maize endosperm $[51,52]$. The rice $E L 2$ was also induced after $24 \mathrm{~h}$ of drought treatment [41]. Similarly, ZmSMR1, ZmSMR5, ZmSMR11 and ZmSMR12 were upregulated by salt and drought stresses, indicating that they may play important roles in enhancing tolerance to salt and drought stresses in maize seeding development. In general, the functions of $Z m S M R$ genes are diverse and are strongly associated with response to abiotic conditions, although the underlying regulatory mechanisms may be complex. A followup study on how to utilize these functions in improving resistance in maize may be necessary.

In summary, the distribution, structure, and phylogenetic relationships of members of the $\mathrm{ZmSMR}$ protein family were comprehensively analyzed in the present study. In addition, these characteristics of $Z m S M R$ genes were correlated with the development of maize endosperm and tissue expression in response to abiotic stresses. These results may improve our understanding of the role of the ZmSMR family in maize and should lay the foundation for future research.

\section{Conclusions}

Twelve putative ZmSMRs were identified by comparison of the SIM/SMR aa sequence in Arabidopsis to the maize genome. The genes were divided into four groups via phylogenetic analysis. Conserved domains consisted of six motifs that were predicted and that supported the clustering results. Four motifs were reported to be associated with the regulation of replication. The temporal expression characteristics of the endosperm development of maize seeds indicate that the ZmSMR gene is negatively correlated with grain filling but that it can initiate the start of endoreduplication at $5 \mathrm{DAP}$ and maintain the high ploidy of endosperm cells at $96 \mathrm{C}$ during 20 and 25 DAP. In addition, ZmSMR9/11/12 may regulate the initiation of endoreplication of endosperm central cells. ZmSMR2/10 may be mainly responsible for the endoreplication regulation of outer endosperm or aleurone cells. In addition, ZmSMR9/11/12 were upregulated under $\mathrm{ABA}$ stress, indicating that they may regulate endoreduplication and adaptation to abiotic stresses, either directly or indirectly. The ZmSMR genes were highly expressed in the ears and tassels, as shown by tissue-specific analysis, indicating that they might be involved in regulating the development of the reproductive organs. These results may provide valuable information for future studies of the function of the SMR family in maize.

\section{Methods \\ Identification of SMR genes in maize}

To identify the $S M R$ genes in the B73 reference genome (RefGen_v4), we used the reported Arabidopsis SIM/ SMR amino acid sequences [48] as queries in a BLASTP search against all maize proteins downloaded from the Phytozome database using an e-value of 1 e- 5 and identity of $50 \%$ as thresholds. The keywords "maize SIM" and "maize SMR" were used as queries to search against the NCBI protein database. The BLASTP and database search hits were compared and parsed by manual editing. Furthermore, a BLASTN of its own genome was performed in maizeGDB to find possible missing gene models. A self-BLAST of these sequences was also performed to remove redundant sequences, and then the remaining sequences were submitted to the NCBI-CDD web server to confirm the presence and integrity of the conserved domains. After manual correcting, the 
putative ZmSMR proteins were obtained. Then the Expasy ProtParam tool (https://web.expasy.org/protparam/) and Softberry-ProtComp Version 9.0 (http:// www.softberry.com/berry.phtml) were used to determine the physicochemical parameters and subcellular localization of the maize SMR proteins, respectively.

\section{Regulatory elements in the promoter region of ZmSMRs}

A 1.5-kb DNA sequence upstream of the initiation codon (ATG) of each $Z m S M R$ was downloaded from the Phytozome database [53]. The transcription start site was designated as +1 . The elements in the promoter fragments (from $-1500 \mathrm{bp}$ to $+1 \mathrm{bp}$ ) of the $Z m S M R$ genes were identified using the PlantCARE (http://bioinformatics.psb.ugent.be/webtools/plantcare/html/) [53, 54], PlantPan 2.0 (http://plantpan2.itps.ncku.edu.tw) [55] and RegSite Database of Plant Regulatory Elements Softberry online program (http://www.softberry.com/ berry.phtml) $[56,57]$.

\section{Identification of syntenic genes}

The syntenic relationships of the maize genes were identified by comparing maize B73 genome sequences using the SynMap [58] utility of the CoGe website (https:// genomevolution.org/coge/). The syntenic genes were detected using CDS data with default settings except for the Quota Align Merge algorithm, and the final syntenic gene-set output with GEvo links was downloaded for further analysis [59].

\section{Phylogenetic analysis and conserved motifs analysis}

Multiple sequence alignment of the full-length protein sequences of ZmSMRs was performed using ClustalW as integrated into MEGA 7.0 with default parameters. The phylogenetic reconstruction was done with MEGA 6.0 using the Neighbor-joining method with 1000 bootstrap replicates [60]. To study the phylogenetic relationship of SMR proteins in Z. mays, A. thaliana, O. sativa, S. bicolor, S. italica, B. distachyon, G. max, P. trichocarpa and P. patens, full-length SMR aa sequences were retrieved from the NCBI and Phytozome databases. In addition, a BLAST of its own genome was performed for these acquired genes to find possible missing gene models. Multiple sequence alignment was performed, and an unrooted tree was plotted as described previously. In addition, to further examine the diversity of motif compositions in the putative ZmSMR proteins, multiple expectation maximization for motif elicitation (MEME) online search software [61] was used to predict the conserved motifs in these proteins. The maximum number of motifs was set to six [62].
Sampling of seeds at different DAP and grain filling rates The maize ( $Z$. mays L.) inbred line B73 was provided by the Key Laboratory of Biology and Genetic Improvement of Maize in Arid Areas of the Northwest Region, Ministry of Agriculture, College of Agronomy, Northwest A\&F University. The lines were grown in the field in the summer of 2012 in Yangling, Shaanxi, China. Ears were self-pollinated on the same day. Seed endosperm at the same position in maize ears was collected every 5 days from 0 to 30 DAP (5 DAP for whole seeds), frozen in liquid nitrogen, and then stored in a $-80^{\circ} \mathrm{C}$ freezer. RNA was isolated from a portion of the seeds and used in testing the relative expression of SMR genes during the development of maize endosperm. The rest of the seeds were kept at $105^{\circ} \mathrm{C}$ for half an hour to deactivate and were then dried to a constant weight at $80^{\circ} \mathrm{C}$. The grain-filling rate was calculated by dividing the increment of hundred-grain weight by the number of days and kernels between two adjacent grain filling stages [16]. Three biological replicates were performed in the qRT-PCR analysis.

\section{Plant growth conditions and stress treatments}

The maize inbred line B73 seeds were immersed in 10\% $\mathrm{H}_{2} \mathrm{O}_{2}$ for 30 min to disinfect and were then treated with $3 \% \mathrm{CaSO} 3$ for $3 \mathrm{~h}$ to promote germination. Treated seeds were grown in Hoagland's solution in a greenhouse under a 14-h light and 10-h dark cycle at $23 \pm$ $1{ }^{\circ} \mathrm{C}$. Leaves were collected from maize seedlings at the three-leaf stage and were then used in the expression profiling of $Z m S M R$ genes under ABA, heat, cold, salt, and drought stresses. For heat and cold stress, the maize seedlings were placed in $42{ }^{\circ} \mathrm{C}$ and $4{ }^{\circ} \mathrm{C}$ environments, respectively. The maize seedlings were immersed in a $100 \mu \mathrm{M}$ ABA solution for hormone treatment. For these three stresses, the leaves from the seedlings were collected after 2, 6, 12, and $24 \mathrm{~h}$. The solutions for salt and drought treatments were prepared by adding $\mathrm{NaCl}(200$ $\mathrm{Mm} / \mathrm{L})$ and PEG $[20 \%(\mathrm{~m} / \mathrm{v})]$ to full-strength Hoagland's solution, respectively. Both treatments lasted $12 \mathrm{~h}, 24 \mathrm{~h}$, $48 \mathrm{~h}$, and $72 \mathrm{~h}$. Control check (CK) seedlings were kept in the unstressed growth conditions. Three biological replicates were performed in the qRT-PCR analysis.

\section{RNA isolation and quantitative real-time PCR}

Total RNA was extracted using the TaKaRa MiniBEST Plant RNA Extraction Kit (Takara, Dalian, China). RNase-free DNase-I was used to remove any contaminating genomic DNA in the solution. First-strand cDNA synthesis was conducted using a FastQuant RT Kit (TIANGEN, Beijing, China) according to the manufacturer's instructions. The qRT-PCR analysis of all 12 $Z m S M R s$ was performed using primers that were designed according to the ZmSMRs' sequences and using 
an NCBI Primer-BLAST online instrument (Additional file 11). The amplification products were controlled within a size range of $130 \mathrm{bp}$ to $250 \mathrm{bp}$ (Additional file 11). The maize actin (Zm00001d013873) gene was used as an internal control. All primers were synthesized by Sangon Biotech Company. The qRT-PCR assays were performed in optical 96-well plates with three technical replicates using the BIO-RAD CFX96 Detection System (Bio-Rad, CA, USA). Each reaction was performed in $20 \mu \mathrm{L}$ of a SuperReal Premix Plus (SYBR Green) reaction mixture (TIANGEN, Beijing, China), following the manufacturer's instructions. The relative expression ratio of each gene was calculated using the $2^{-\triangle \Delta C T}$ method [63].

\section{Supplementary information}

Supplementary information accompanies this paper at https://doi.org/10. 1186/s12862-020-01619-2.

\section{Additional file 1. Gene ID and Sequence of ZmSMRs.}

Additional file $\mathbf{2}$ The numbers and locations of regulatory cis-acting elements included in ZmSMR genes. (a) The number of genes for every cisacting element. E2Fa/b, endoreduplication starting element (En); 5'UTR Py-rich stretch (5UTR), cis-acting element conferring high transcription levels; Ry-element, cis-acting regulatory element involved in seed-specific regulation; Skn-1_motif, cis-acting regulatory element required for endosperm expression; MBS/ MBSI, MYB binding site involved in droughtinducibility; HSE, cis-acting element involved in heat stress responsiveness; LTR, cis-acting element involved in low-temperature responsiveness; ABRE/CE3, cis-acting element involved in ABA responsiveness. (b) The location of these regulatory cis-acting elements in ZmSMR gene promoters. The elements are distinguished by different colors. The upstream sequence scale is shown above, and the consensus sequences of each component are also shown at the bottom of the figure. Detailed locations, functions, and numbers can be found in Additional file 3 .

Additional file $\mathbf{3}$ The number of various cis-acting elements in ZmSMR promoters.

Additional file 4. Genomic and intergenomic synteny SMR gene pairs in multiple species.

Additional file 5. Alignment results of genes on the same branch of the ZmSMR genes phylogenetic tree.

Additional file 6. Overview of conserved motifs of ZmSMR genes identified through MEME analysis.

Additional file $\mathbf{7}$ List of the SMR protein sequences in maize, Arabidopsis thaliana, rice, Brachypodium distachyod, millet, sorghum, soybean and Populus trichocarpa.

Additional file 8 Number of SMR in maize, Brachypodium distachyon, barley, millet, sorghum, soybean, Populus trichocarpa, rice, Arabidopsis.

Additional file $\mathbf{9}$ Orthology genes of maize in Sorghum bicolor, Setaria italica, Oryza sativa and Brachypodium distachyon.

Additional file 10. The number of haploid genomes in different ploidy level at different times after pollination.

Additional file $\mathbf{1 1}$ Primers for qRT-PCR to investigate the ZmSMR genes.

\section{Abbreviations}

SIM: SIAMESE; CKI: Cell cycle kinase inhibitor; SMR: SIAMESE-RELATED; ZmSMR: Zea mays L. SIAMESE-RELATED; DAP: Days after pollination; CDKs: Cyclin-dependent kinases; CYC: Cyclin; CYCDs: D-type cyclins; DE: Decision Element; HSE: Heat shock element; LTR: Low temperature responsive element; ABREs: ABA-responsive elements

\section{Acknowledgments}

We thank Dr. Xiaojun Nie for his assistance in writing.

\section{Authors' contributions}

DWG, ZQZ and JZQ designed the research. ZQZ and FFL performed the research. ZQZ, JZQ and SLL performed bioinformatics analysis, including gene identification and GPCR data analysis. ZQZ, STX, RHZ and JQX wrote the original draft. All authors read and approved the final manuscript.

\section{Funding}

This work was supported by National Natural Science Foundation of China (31371626) and Sci-Tech Project of Shaanxi Province (2015KTZDNY01-01-01). We are also grateful for the support of Shaanxi Agricultural Department 2017 Provincial Modern Crop Seed Industry Project (20171010000004). The funding body played an important role in the design of the study and collection, analysis, interpretation of data and in writing the manuscript.

Availability of data and materials

The datasets supporting the conclusions of this article are included within the article and its additional files.

Ethics approval and consent to participate

This article does not contain any studies with human participants or animal performed by any of the authors.

\section{Consent for publication}

Not applicable.

\section{Competing interests}

The authors declare that there are no competing interests.

Received: 7 July 2019 Accepted: 29 April 2020

Published online: 29 July 2020

References

1. Dewitte W, Murray JA. The plant cell cycle. Annu Rev Plant Biol. 2003;54: 235-64.

2. Ramirez-Parra E, Desvoyes B, Gutierrez C. Balance between cell division and differentiation during plant development. Int J Dev Biol. 2005;49(5-6):46777.

3. Edgar BA, Orr-Weaver TL. Endoreplication cell cycles more for less. Cell. 2001:105:297-306.

4. Larkins BA, Dilkes BP, Dante RA, Coelho CM. Investigating the hows and whys of DNA endoreduplication. J Exp Bot. 2001:52(355):183-92.

5. D'Amato F. Role of polyploidy in reproductive organs and tissues. In: Johri BM, editor. Embryology of angiosperms. Berlin: Springer; 1984. p. 519-66.

6. Inze D, De Veylder L. Cell cycle regulation in plant development. Annu Rev Genet. 2006:40:77-105.

7. Gendreau E, Traas J, Desnos T, Grandjean O, Caboche M, Hofte H. Cellular basis of hypocotyl growth in Arabidopsis thaliana. Plant Physiol. 1997;114(1): 295-305.

8. Kudo N, Kimura Y. Nuclear DNA endoreduplication during petal development in cabbage: relationship between ploidy levels and cell size. J Exp Bot. 2002;53(371):1017-23.

9. Kladnik A, Chourey PS, Pring DR, Dermastia M. Development of the endosperm of Sorghum bicolor during the endoreduplication-associated growth phase. J Cereal Sci. 2006;43(2):209-15.

10. Cheniclet C, Rong WY, Causse M, Frangne N, Bolling L, Carde JP, et al. Cell expansion and endoreduplication show a large genetic variability in pericarp and contribute strongly to tomato fruit growth. Plant Physiol. 2005; 139(4):1984-94.

11. Jakoby M, Schnittger A. Cell cycle and differentiation. Curr Opin Plant Biol. 2004;7(6):661-9.

12. Melaragno JE, Mehrotra B, Coleman AW. Relationship between Endopolyploidy and cell size in epidermal tissue of Arabidopsis. Plant Cell. 1993:5(11):1661-8

13. Ceccarelli M, Santantonio E, Marmottini F, Amzallag GN, Cionini PG. Chromosome endoreduplication as a factor of salt adaptation in Sorghum bicolor. Protoplasma. 2006;227(2-4):113-8. 
14. Engelen-Eigles G, Jones RJ, Phillips RL. DNA endoreduplication in maize endosperm cells: the effect of exposure to short-term high temperature. Plant Cell Environ. 2000;23(6):657-63.

15. Barow M. Endopolyploidy in seed plants. BioEssays. 2006;28(3):271-81.

16. Jin X, Fu Z, Lv P, Peng Q, Ding D, Li W, et al. Identification and characterization of microRNAs during maize grain filling. PLoS One. 2015; 10(5):e0125800.

17. Sabelli PA, Leiva-Neto JT, Dante RA, Hong N, Larkins BA. Cell cycle regulation during maize endosperm development. Maydica. 2005;50:485-96.

18. Kowles RV, Phillips RL. Endosperm development in maize. Int Rev Cytol. 1988;112:97-136.

19. Sun Y, Flannigan BA, Setter TL. Regulation of endoreduplication in maize (Zea mays L.) endosperm. Isolation of a novel B1-type cyclin and its quantitative analysis. Plant Mol Biol. 1999;41(2):245-58.

20. Dante RA. Characterization of cyclin-dependent kinases and their expression in developing maize endosperm [electronic resource]. Tucson: The University of Arizona; 2005

21. Sun Y, Dilkes BP, Zhang C, Dante RA, Carneiro NP, Lowe KS, et al. Characterization of maize (Zea mays L.) Wee1 and its activity in developing endosperm. Proc Natl Acad Sci U S A. 1999;96(7):4180-5.

22. Coelho CM, Dante RA, Sabelli PA, Sun Y, Dilkes BP, Gordon-Kamm WJ, et al. Cyclin-dependent kinase inhibitors in maize endosperm and their potential role in endoreduplication. Plant Physiol. 2005;138(4):2323-36.

23. Sabelli PA, Dante RA, Leiva-Neto JT, Jung R, Gordon-Kamm WJ, Larkins BA. RBR3, a member of the retinoblastoma-related family from maize, is regulated by the RBR1/E2F pathway. Proc Natl Acad Sci U S A. 2005;102(37): 13005-12.

24. Sabelli PA, Larkins BA. Grasses like mammals redundancy and compensatory regulation within the retinoblastoma protein family. Cell Cycle. 2006;5(4): 352-5.

25. Kim JC, Laparra H, Calderon-Urrea A, Mottinger JP, Moreno MA, Dellaporta SL. Cell cycle arrest of stamen initials in maize sex determination. Genetics. 2007;177(4):2547-51

26. Larkin JC, Brown ML, Schiefelbein J. How do cells know what they want to be when they grow up? Lessons from epidermal patterning in Arabidopsis. Annu Rev Plant Biol. 2003;54:403-30.

27. Bramsiepe J, Wester K, Weinl C, Roodbarkelari F, Kasili R, Larkin JC, et al. Endoreplication controls cell fate maintenance. PLoS Genet. 2010;6(6):e1000996.

28. Walker JD, Oppenheimer DG, Concienne J, Larkin JC. SIAMESE, a gene controlling the endoreduplication cell cycle in Arabidopsis thaliana trichomes. Development. 2000;127(18):3931-40.

29. Churchman ML, Brown ML, Kato N, Kirik V, Hulskamp M, Inze D, et al. SIAMESE, a plant-specific cell cycle regulator, controls endoreplication onset in Arabidopsis thaliana. Plant Cell. 2006;18(11):3145-57.

30. Rogers $S$, Wells $R$, Rechsteiner M. Amino acid sequences common to rapidly degraded proteins: the PEST hypothesis. Science (New York, NY). 1986; 234(4774):364-8

31. He J, Wang D, Zhang J, Wang Y. Functional analysis of the fruit-specific promoter of VqSTS6 from the Chinese wild grape, Vitis quinquangularis. Agri Gene. 2016;1:38-45.

32. Lescot $M$, Déhais $P$, Thijs $G$, Marchal $K$, Moreau $Y$, Van de Peer $Y$, et al. PlantCARE, a database of plant cis-acting regulatory elements and a portal to tools for in silico analysis of promoter sequences. Nucleic Acids Res. 2002;30(1):325-7

33. Frolov $\mathrm{V}$. M: functional antagonism between E2F family members. Genes Dev. 2001;15(16):2146-60.

34. Zhang J, Zhang X, Wang Y, Hou H, Qian Y. Characterization of sequence elements from Malvastrum yellow vein betasatellite regulating promoter activity and DNA replication. Virol J. 2012;9(234):2-9.

35. Zhang ZY, Zhao J, Hu Y, Zhang TZ. Isolation of GhMYB9 gene promoter and characterization of its activity in transgenic cotton. Biologia Plantarum Vol. 2015;59(4):629-36

36. Medina J, Barques M, Terol J, Pe'rez-Alonso M, Salinas J. The Arabidopsis CBF gene family is composed of three genes encoding AP2 domaincontaining proteins whose expression is regulated by low temperature but not by abscisic acid or dehydration. Plant Physiol. 1999;119(2):463-9.

37. Barcala M, García A, Cubas P, Almoguera C, Jordano J, Fenoll C, et al. Distinct heat-shock element arrangements that mediate the heat shock, but not the late-embryogenesis induction of small heat-shock proteins, correlate with promoter activation in root-knot nematode feeding cells. Plant Mol Biol. 2007;66(1-2):151-64.
38. Hobo T, Asada M, Kowyama Y, Hattori T. ACGT-containing abscisic acid response element (ABRE) and coupling element 3 (CE3) are functionally equivalent. Plant J. 1999;19(6):679-89.

39. Freeling M. Bias in plant gene content following different sorts of duplication: tandem, whole-genome, segmental, or by transposition. Annu Rev Plant Biol. 2009;60:433-53.

40. SanMiguel P, Gaut BS, Tikhonov A, Nakajima Y, Bennetzen JL. The paleontology of intergene retrotransposons of maize. Nat Genet. 1998; 20(1):43-5.

41. Peres A, Churchman ML, Hariharan S, Himanen K, Verkest A, Vandepoele K, et al. Novel plant-specific cyclin-dependent kinase inhibitors induced by biotic and abiotic stresses. J Biol Chem. 2007;282(35):25588-96.

42. De Veylder L, Beeckman T, Beemster GT, Krols L, Terras F, Landrieu I, et al. Functional analysis of cyclin-dependent kinase inhibitors of Arabidopsis. Plant Cell. 2001:13(7):1653-68.

43. Kumar N, Harashima H, Kalve S, Bramsiepe J, Wang K, Sizani BL, et al. Functional conservation in the SIAMESE-RELATED family of cyclindependent kinase inhibitors in land plants. Plant Cell. 2015;27(11):3065-80

44. Kay BK, Williamson MP, Sudol M. The importance of being proline: the interaction of proline-rich motifs in signaling proteins with their cognate domains. FASEB J. 2000;14(2):231-41.

45. Sabelli PA, Larkins BA. The contribution of cell cycle regulation to endosperm development. Sex Plant Reprod. 2009;22(4):207-19.

46. Cao J. Analysis of the Prefoldin gene family in 14 plant species. Front Plant Sci. 2016;7:317.

47. Singh AK, Sharma V, Pal AK, Acharya V, Ahuja PS. Genome-wide organization and expression profiling of the NAC transcription factor family in potato (Solanum tuberosum L.). DNA Res. 2013;20(4):403-23.

48. Yi D, Alvim Kamei CL, Cools T, Vanderauwera S, Takahashi N, Okushima Y, et al. The Arabidopsis SIAMESE-RELATED cyclin-dependent kinase inhibitors SMR5 and SMR7 regulate the DNA damage checkpoint in response to reactive oxygen species. Plant Cell. 2014;26(1):296-309.

49. Grafi G, Larkins BA. Endoreduplication in maize Endosperm_involvement of $M$ phase--promoting factor inhibition and induction of $S$ phase--related kinases. Science. 1995;269(5228):1262-4.

50. Nemhauser JL, Hong F, Chory J. Different plant hormones regulate similar processes through largely nonoverlapping transcriptional responses. Cell. 2006:126(3):467-75.

51. Setter $T L$, Flannigan BA. Water deficit inhibits cell division and expression of transcripts involved in cell proliferation and endoreduplication in maize endosperm. J Exp Bot. 2001;52(360):1401-8.

52. Artlip TS, Madison JT, Setter TL. Water deficit in developing endosperm of maize_cell division and nuclear DNA endoreduplication. Plant Cell Environ. 1995;18(9):1034-40.

53. Jiang Y, Duan Y, Yin J, Ye S, Zhu J, Zhang F, et al. Genome-wide identification and characterization of the Populus WRKY transcription factor family and analysis of their expression in response to biotic and abiotic stresses. J Exp Bot. 2014;65(22):6629-44.

54. Zhao P, Wang D, Wang R, Kong N, Zhang C, Yang C, et al. Genome-wide analysis of the potato Hsp20 gene family: identification, genomic organization and expression profiles in response to heat stress. BMC Genomics. 2018;19(1):61.

55. Chow $\mathrm{CN}$, Zheng HQ, Wu NY, Chien $\mathrm{CH}$, Huang HD, Lee TY, et al. PlantPAN 2.0: an update of plant promoter analysis navigator for reconstructing transcriptional regulatory networks in plants. Nucleic Acids Res. 2016;44(D1): D1154-60.

56. Solovyev W, Shahmuradov IA, Salamov AA. Identification of promoter regions and regulatory sites. Methods Mol Biol. 2010;674:57-83.

57. Shahmuradov IA, Solovyev W. Nsite, NsiteH and NsiteM computer tools for studying transcription regulatory elements. Bioinformatics. 2015; 31(21):3544-5.

58. Lyons E, Pedersen B, Kane J, Freeling M. The value of nonmodel genomes and an example using SynMap within CoGe to dissect the Hexaploidy that predates the Rosids. Trop Plant Biol. 2008;1(3-4):181-90.

59. Miao Z, Han Z, Zhang T, Chen S, Ma C. A systems approach to a spatiotemporal understanding of the drought stress response in maize. Sci Rep. 2017;7(1):6590.

60. Tamura K, Peterson D, Peterson N, Stecher G, Nei M, Kumar S. MEGA5: molecular evolutionary genetics analysis using maximum likelihood, evolutionary distance, and maximum parsimony methods. Mol Biol Evol. 2011;28(10):2731-9. 
61. Bailey TL, Williams N, Misleh C, Li WW. MEME: discovering and analyzing DNA and protein sequence motifs. Nucleic Acids Res. 2006;34:W369-73 Web Server issue.

62. Bailey TL, Boden M, Buske FA, Frith M, Grant CE, Clementi L, et al. MEME SUITE: tools for motif discovery and searching. Nucleic Acids Res. 2009;37: W202-8 Web Server issue.

63. Livak KJ, Schmittgen TD. Analysis of relative gene expression data using real-time quantitative PCR and the 2(-Delta Delta $C(T))$ method. Methods. 2001;25(4):402-8.

\section{Publisher's Note}

Springer Nature remains neutral with regard to jurisdictional claims in published maps and institutional affiliations.

Ready to submit your research? Choose BMC and benefit from:

- fast, convenient online submission

- thorough peer review by experienced researchers in your field

- rapid publication on acceptance

- support for research data, including large and complex data types

- gold Open Access which fosters wider collaboration and increased citations

- maximum visibility for your research: over $100 \mathrm{M}$ website views per year

At $\mathrm{BMC}$, research is always in progress.

Learn more biomedcentral.com/submissions 